\title{
An Efficient Framework for Macula Exudates Detection in Fundus Eye Medical Images
}

\author{
Noha A. El-Hag \\ Communications and Electronics \\ Department \\ Faculty of Electronic \\ Engineering,Manoufia University: \\ Menouf, Egypt \\ nohaeng940@yahoo.com \\ El-Sayd M. El-Rabaie \\ Communications and Electronics \\ Department \\ Faculty of Electronic \\ Engineering,Manoufia University: \\ Menouf, Egypt \\ srabie1@yahoo.com
}

\author{
Walid El-Shafie \\ Communications and Electronics \\ Department \\ Faculty of Electronic \\ Engineering,Manoufia University: \\ Menouf, Egypt \\ wngwalidelshafie@yahoo.com \\ Adel S. El-Fishawy \\ Communications and Electronics \\ Department \\ Faculty of Electronic \\ Engineering,Manoufia University: \\ Menouf, Egypt, \\ aelfishawy@hotmail.com
}

\author{
Ghada M. El-Banby \\ Automatic Control Department \\ Faculty of Electronic \\ Engineering,Manoufia University: \\ Menouf, Egypt \\ ghadabanby@yahoo.com
}

Fathi I. Abd El-Samie
Communications and
ElectronicsDepartment
Faculty of Electronic
Engineering,
Menuufia Univeristy:
Menof, Egypt
fathi_sayed@yahoo.com

\begin{abstract}
This paper presents a computer-based framework for the segmentation of medical eye images. Also, the proposed framework achieves the detection of exudates in medical eye images for better diagnosis of maculopathy disease. The proposed framework begins with fuzzy image enhancement of eye images for contrast enhancement in order to enhance the objects representation of the images. After that, the segmentation process is performed to determine the optic disc and blood vessels to remove them. The next step is detecting the region of interest edges in exudates. A gradient process is also performed on the image and the histogram of gradient is evaluated. Accumulative histogram is further generated for discrimination between image with and without exudates. A threshold histogram curve is generated based on predefined images with and without exudates for classification of images in the testing phase. The simulation results prove that the proposed framework has an appreciated performance.
\end{abstract}

Keywords-Diabetic Maculpathy, Exudates, Gaussian gradient, Fuzzy Technique, Macula, Gaussian Gradient

\section{INTRODUCTION}

The anatomy of the eye retina includes optic disc, blood vessels, vascular network and macula. The macula is the tool that handles color and reading vision .In maculpathy disease, some exudates appear in the macula region. A good diagnosis of maculpathy disease is achieved through the localization of the exudates. Exudates are yellow flecks made up of lipid residues leakage damaged capillaries. It is look like yellow-white things in retina. Several attempts have been performed for exudates localization in retinal images [1-2].

Pre-processing is a required step for any diagnosis process performed on conferral microscopy images. Different methods have been implemented for pre-processing of eye images to improve the contrast of these images prior to the segmentation process. One of the most popular tools for contrast enhancement of images is contrast limited adaptive histogram equalization (CLAHE). It works locally on blocks and puts certain limits on the pixel levels.In [3], the authors utilized the CLAHE technique for enhancement of green channel of retinal images. After that, the morphological operation is performed to shape objects regularly. Support vector machine is used for classification process to help for discovering the disease.

In [4], another attempt for the detection of diabetic maculopathy disease was performed. This algorithm performs pre-processing for retinal images. After that image segmentation is performed to split the image pixels based on image feature. The macula and its exudates can be detected by using morphological operations. The attempt worked on two types of the disease (Non-clinically, clinically significant maculpathy) and usual eye. The classification is performed by two methods ANN and PNN. The sensitivity for ANN and PNN is 96.67 and 28.33, the specificity is 100 .

In [5], a system for detection the retinal image exudates is introduced. Some processing algorithms are applied. Background homogenization works in green channel. Non uniform illumination results from intensity variation of the image background so that shade corrected image is used .Adaptive noise removal filter is used and worked in small sized neighborhood and noise variance. Adaptive global thresholding process is performed to extract its brightness pixels. Feature extraction regions are previously determined by performing a set of features mathematically. Gaussian and its derivatives up to order 2 at five different scales are used to determine the features. Supervised classification method is performed to detect exudates regions.

In [6], this method is applied for optic disc detection in low noisy contrast. A red channel is used to detect the optic disc. The image is filtered to eliminate large gray level variations and closing morphological operation is used. Optic disc is 
localized exploiting its high gray level variations as well as Threshold area is used to localize optic disc. To detect the vascular tree green channel is used. Simple Gaussian filter is performed to remove the noise. Opening morphological operation and top hat transform are performed.

Fuzzy technique aims to enhance the visual quality of an image by strengthening edges and smoothing flat areas. Medical image analysis is required for good contrast images. In [7], a method for image enhancement is developed. Fuzzy techniques consist of three stages of fuzzification, modification of the membership, and defuzzification. For fuzzification the image is converted from spatial domain to fuzzy domain, then for modification of the membership the intensification operator is used to modify image from low quality to high quality. Finally, by applying defuzzification method the image is transformed back to spatial domain.

The utilization of fuzzy concept has been found popular in retinal images processing. It is used for the enhancement of the retinal images. In [8], a fuzzy based algorithm for retinal images enhancement and segmentation is presented. It is based on fuzzy morphological operations including closing process for blood vessels segmentation. A double threshold operator is used with different values. This algorithm achieved an accuracy of 95.37 , a sensitivity of 72.26 , and a specificity of 97.81 .

In [9], the authors developed an algorithm for enhancing the image that the entropy value is applied in the neighborhood window as a factor to measure the intended pixel level to be an edge point. The fuzzy local contrast is also introduced and a certain threshold is set to judge where intended local contrast of pixels in neighboring region should be improved. Adaptive choosing of the point center of neighborhood in fuzzy contrast enhancement is built up.

In [10], the authors developed a fuzzy gray scale enhancement system for images that have low quality. The lack of the contrast of image is occurred by inadequate lighting during image capturing and non-uniform illumination of the image is resulted. The fuzzy gray scale image improving method is applied by maximizing fuzzy measures contained in the image, after that the image is improving, and then the modification of membership is implemented by using saturation operator and transformation of the power-law. Membership function is used for representation the degree of brightness or whiteness in gray level images.

This paper presents a framework for the segmentation of medical eye images. Also, the proposed framework achieves the detection of exudates in medical eye images for the diagnosis of maculopathy disease. The paper is organized as follows. Section 2 describes the proposed steps of data acquisition and processing for identification of the diabetic maculopathy. Section 3 introduces the simulation and comparative results. Section 4 gives the paper conclusions

\section{THE PROPOSED DATA ACQUISTION AND PROCESSING FRAMEWORK}

In the proposed framework, the eye image is firstly converted to a green channel component .Image processing techniques are used to determine the optic disc, macula and exudates on it. Exudates with histogram of gradient is used for detection the exudates in macula region. Figure 1 shows the block diagram of the proposed system for detecting the diabetic maculopathy stages. The proposed system will be discussed in-detail in the following section.

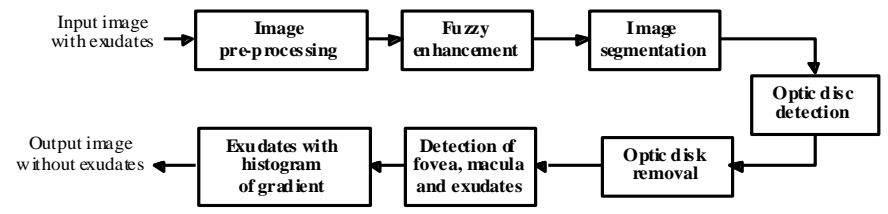

Fig.1the block diagram of detecting the diabetic maculpathy disease.

\section{A. Image Enhancement Based onFuzzy Logic}

The concept of fuzziness has been applied in recent years to achieve contrast enhancement and give image with high contrast. Figure 2 represents the general structure of fuzzy image includes three basic stages: Fuzzification, modification of the membership and defuzzication [11]. The size of the image is $\mathrm{M} \times \mathrm{N}$ and $\mathrm{L}$ a gray levels that can be used for determining a function of image membership. The proposed work uses intensification operator to reduce the fuzziness and to improve the image quality.

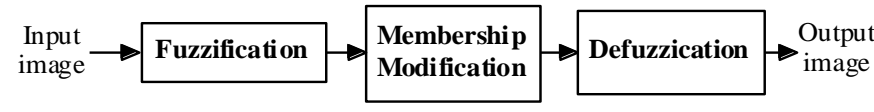

Fig.2Fuzzy Enhancement Technique.

The process of image contrast enhancement process based on fuzzy logic is explained as follows:

\section{- Image Fuzzification}

For an image I, let the gray level at location $(i, j)$ be given by , and the maximum and minimum value of the gray level be given by Xmax and Xmin. The membership function is the membership function is defined as:

$$
\mu_{i j}=\left(x_{i j}\right)-\left(x_{\min }\right) /\left(x_{\max }-x_{\min }\right)
$$

\section{- Modification of Membership}

Membership values are modified using intensifier operators. It converts values of the membership that are higher than 0.5 (default value) to high values and the values of membership that are lower than 0.5 to lower values in a nonlinear method.

$$
\mu_{i j}=\left\{\begin{array}{l}
2 *\left[\mu_{i j}\right]^{2} \mathrm{O} \leq \mu_{i j} \leq 0.5 \\
1-2 *\left[1-\mu_{i j}\right]^{2} \mathrm{O} \leq \mu_{i j} \leq 1
\end{array}\right.
$$




\section{- Image Defuzzication}

Modified gray values are transformed to spatial domain.

$X^{\prime}{ }_{i j}=\underset{\min }{X}-\mu_{i j} *(\underset{\max }{X}-\underset{\min }{X})$

$\boldsymbol{X}{ }_{i j}$ is the required enhanced image obtained after defuzzication process. The aim of our proposed method makes the contrast enhancement more adaptive and more effective [12-15].

\section{B. Image Segmentation}

Image segmentation is a technique splitting image in to parts, called segments. The image is split into several parts based on certain image features. The goal of image segmentation is to divide an image into several parts having similar features. The threshold value is selected 0.95 [1619].Figure 3 shows the image is segmented.

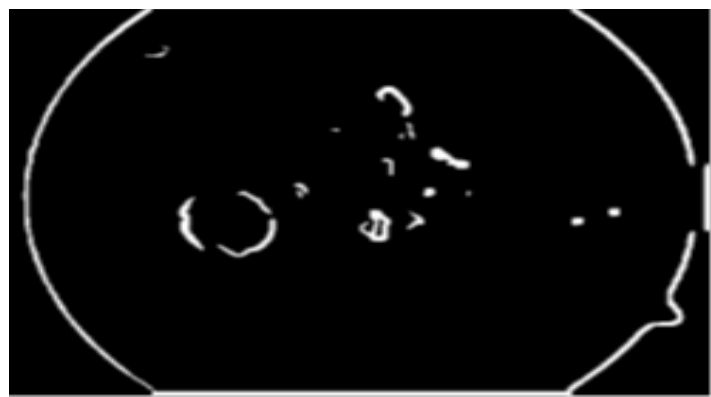

Fig.3. Image Segmentation.

\section{Detection of The Optic Disc}

Optic disc emerges with the same intensity and color to the fundus image. Blood vessels emerge with high contrast as optic disc. Morphological closing operator will aid to delete the vessels which it found in the region. After determining the optic disc a circular mask is built up to overlay the optic disc area. Figure 4 shows the steps outputs of the optic disk detection. Figure 4(a) shows the result after closing operator, (b) the resulting image with thresholding is 0.95 , (c) The location of the maximum of the image in the center of optic disk, (d) the detection of the optic disc and (e) A circular mask is then built up to overlay the optic disc region [20-24].

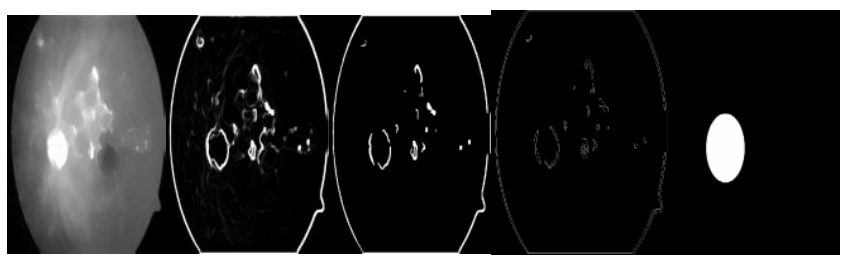

(a)

(b)

(c) (d)

(e)

Fig.4.The output results of optic disc detection: (a) Image after closing, (b) columnwise neighborhood operation, (c) Image segmentation, (d) Optic disc detection, (e) Circular mask on optic disc.

\section{Detection of Exudates}

Exudates look as bright parts in color retinal images. The exudates size and shape change and their borders are mostly inordinate during maculpathy disease. There is a difficulty for determining exudates as a result of a presence optic disc and blood vessels [21]. Morphological operations are used for exudates detection. Erosion, dilation, opening and closing are using for detecting the exudates. This technique uses morphological operations to allow the exudates to be appeared clearly. The exudates are obtained after optic disc removing and circular border. Closing is performed in the image. The exudates have been filled when dilation is applied. The erosion is used to stretch exudates size [22]. Figure 5 described the image after closing and exudates region in the macula.Figure 6 shows the fundus image and the resulted exudates: (a) exudates of normal and abnormal images, (b) normal and abnormal images.

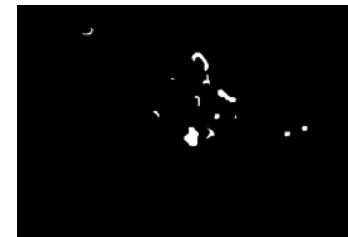

(a)

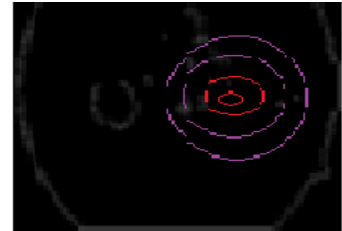

(b)
Fig.5Morphological operation: (a) Morphological closing operation, (b) Exudates in macular

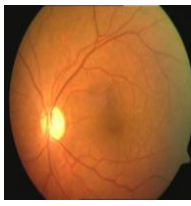

(a)

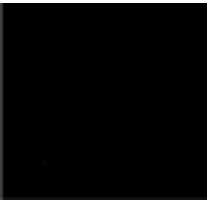

(b)

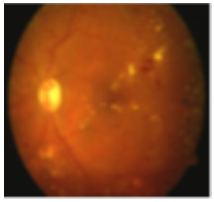

(c)

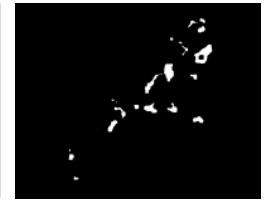

(d)
Fig.6. .An image of the retinal of the eye and its exudates: (a) Exudates of normal image, (b) normal image, (c) Exudates of abnormal image, (d) Abnormal image.

\section{E. Exudates With Histogram Gradient}

The technique counts occurrence of gradient orientation in localized regions of an image detection window. The image is divided in to cells. Each cell computes the histogram of gradient orientations. Cumulative Histogram is generated for each cell. A histogram based strategy is helped for maculpathy detection. The histogram of the edges is not enough to detect the maculpathy, but a cumulative histogram is generated for each case. Certain scale of cumulative histogram values is selected to distinguish between normal and abnormal images. Gaussians gradient is used to detect the disease. The basic idea is applying a Gaussian kernel on the image with a certain scale. Consider a gray-scale image as a function $\mathrm{f}(\mathrm{x}, \mathrm{y})$. The following Gaussian kernel is applied on the image [25].

$G(x, y)=1 /\left(2 \pi \sigma^{\wedge} 2\right)$

Applying the derivatives of the Gaussian kernel in both $\mathrm{x}$ and $y$ directions on the image. The derivative of the Gauss kernel in Eq. (6) with $\mathrm{x}$ is given by [26]:

$G_{d x}=\frac{\partial G(x, y)}{\partial x}=\frac{-x G(x, y)}{\sigma^{2}}$

Also, the derivative with y is given by [26]:

$G_{d x}=\frac{\partial G(x, y)}{\partial y}=\frac{-x G(x, y)}{\sigma^{2}}$

Due to the commutative property between the derivative operator and the Gaussian smoothing operator, such scale- 
space derivatives can equivalently be computed by convolving the original image $\mathrm{f}(\mathrm{x}, \mathrm{y})$ with the Gaussian derivative operators $\left[\mathrm{G}_{\mathrm{dx}}, \mathrm{G}_{\mathrm{dy}}\right]$.

This means that the edge map components in both $\mathrm{x}$ and $\mathrm{y}$ directions are given by [27].

$$
\begin{aligned}
G_{d x} & =\otimes f(x, y) \\
D_{f y} & =G_{d y} f(x, y)
\end{aligned}
$$

From Equations. (7) and (8), we deduce that both RD_(f_x ) and D 2 _(f_y ) reveal the image details due to the derivative effect, where $\otimes$ refers to the convolution operation. The proposed approach depends on estimating the details in a common image format as follows [27]:

$$
D_{f_{x y}}=\left|D_{f x}\right|+\left|D_{f y}\right|
$$

Finally, from Eq. (9), we can work on the histogram of $[7$ D)_(f_xy ). Gaussian gradient algorithm has been applied on 60 images .Both the histogram and cumulative histogram have been estimated for all images. Always, there is a difference between the cumulative histogram curves of normal and abnormal images. So, a threshold can be set in the middle of the two curves at a certain bin value. It is possible to distinguish between the exudates of two images by setting a threshold at a certain bin value in the cumulative histogram. Figure (7) shows the block diagram of Gaussian gradient.

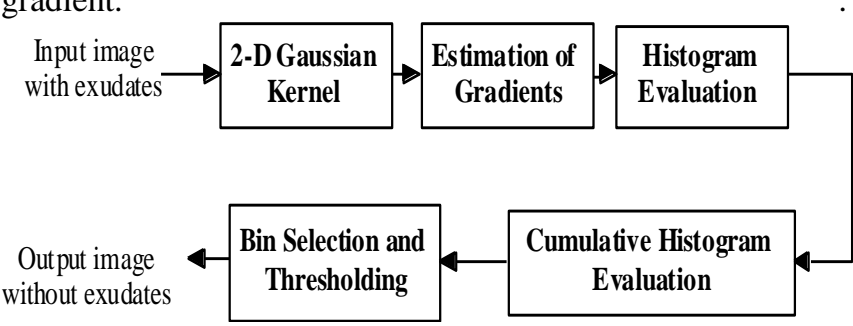

Fig.7The block diagram of the proposed histogram Gaussian Gradient process.

\section{SIMULATION RESULTS}

The proposed algorithm was applied to 60 retinal images (576x720) and performed using matlab 2015a on pc with windows 7professional operating system.After fuzzy technique is implemented the exudates appear obviously for normal and abnormal retinal images that help ophthalmology to detect maculpathy disease. After Gaussian Gradient is also implemented .Using the resulted exudates for detect the normal and abnormal cases by certain scale of commutative histogram. Figure (8) shows the fundus image and its exudates before fuzzy method, (a) the normal image,(b) the exudates of normal image. Figure (9) shows the fundus image and its exudates after fuzzy method,(a) the normal image,(b) the exudates of normal image. Figure 10 shows abnormal image and its exudates before fuzzy method. (a) abnormal image, (b) resulted exudates of abnormal image. Figure 11 shows the abnormal image and the resulted exudates after fuzzy technique. (a) abnormal image ,(b)

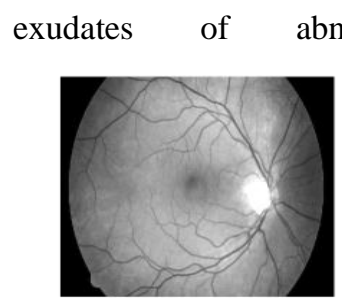

(a)

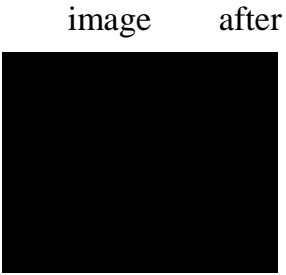

(b)
Fig.8.Original retinal image and its exudates :( a) The original image, (b) Exudates of original image before fuzzy enhancement.

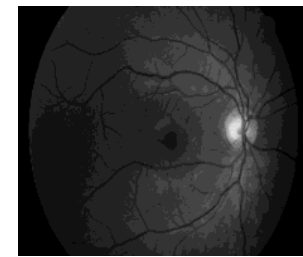

(a)

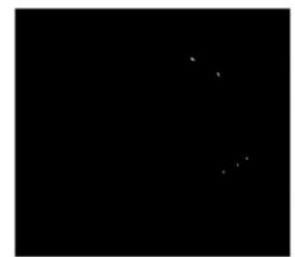

(b)
Fig.9.Reinal image and its exudates: (a) Original normal image,(b)Exudates of original normal image after fuzzy enhancement technique.

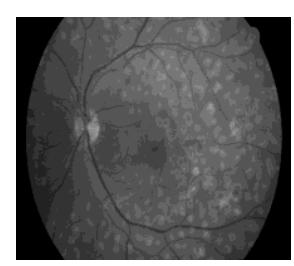

(a)

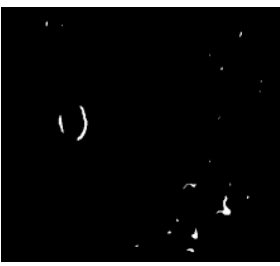

(b)
Fig.10.Retinal image with maculpathy disease and its exudates before fuzzy: (a) Abnormal image, (b) Exudates of abnormal image before fuzzy technique.

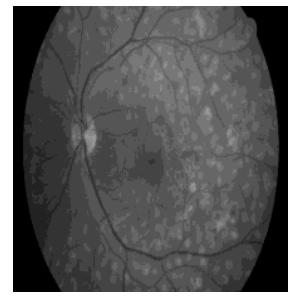

(a)

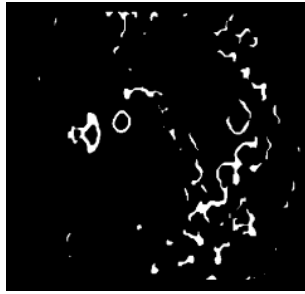

(b)
Fig.11. Enhanced abnormal image and its exudates :( a) Enhanced abnormal image,(b) Exudates of abnormal image after fuzzy enhancement technique.

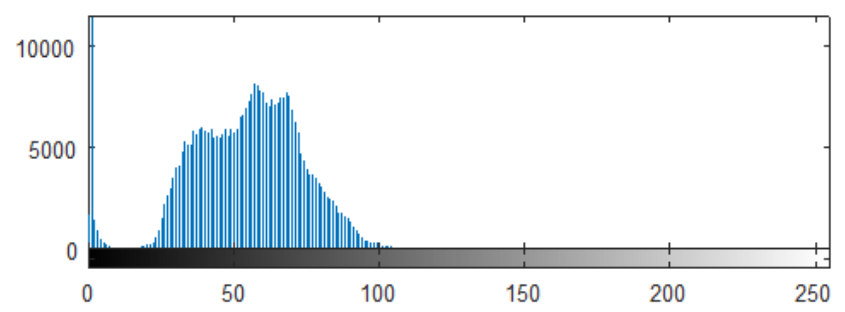

(a) 


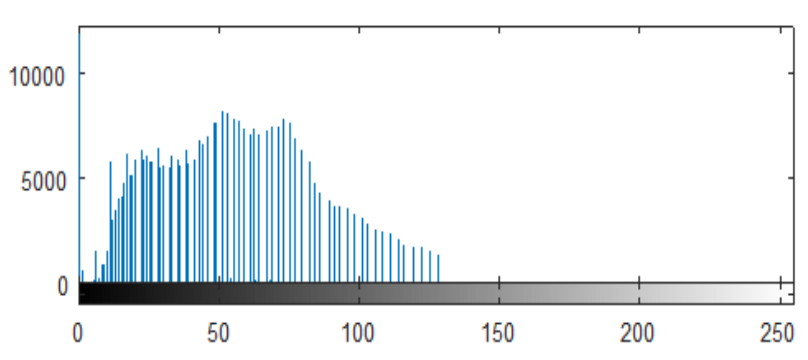

(b)

Fig.12. (a) Histogram of original image shown in Fig.8. (b) Histogram of enhanced normal image shown in Fig. 9.

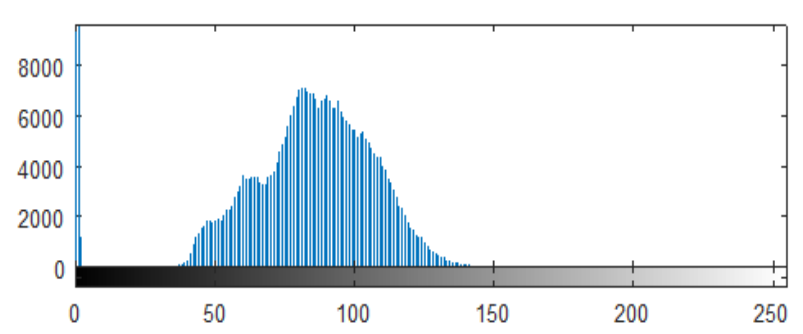

(a)

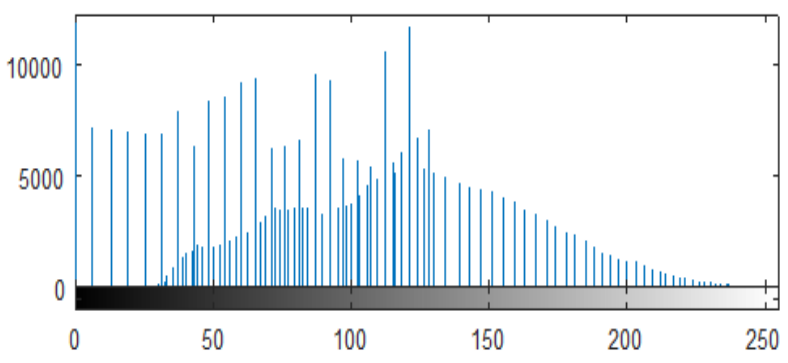

(b)

Fig.13 (a) Histogram of original image shown in Fig .10, (b) Histogram of enhanced abnormal image shown in Fig.11.

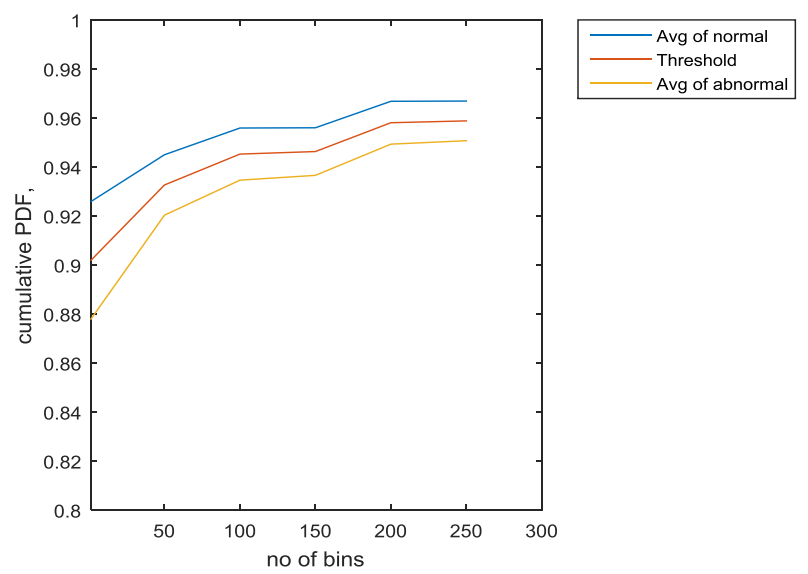

Fig. 14 The cumulative histograms of exudates of the normal and abnormal images before fuzzy enhancement technique.
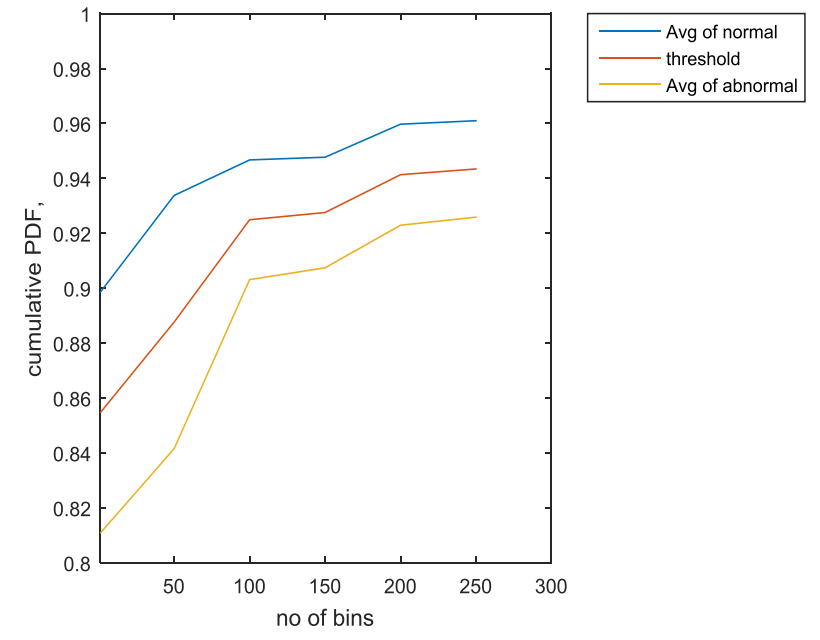

Fig. 15 The cumulative histograms of exudates of the normal and abnormal images after enhancement technique.

Sensitivity is the proportion of patients with disease who test positive. Specificity is the proportion of patients without disease who test negative. The Positive Predictive Accuracy (PPA) is the proportion of patients with positive tests who have disease. The sensitivity, specificity, and Positive Predictive Accuracy (PPA) are determined by the following formulas, where TP refers to True Positive case, TN refers to True Negative case, FP refers to False Positive case, and FN refers to False Negative case. The results of the sensitivity, specificity, and PPA are computed before and after fuzzy as introduced in Table 1.

$$
\begin{aligned}
& \text { Sensitivity }=\mathrm{TP} /(\mathrm{TP}+\mathrm{FN}) \\
& \text { Specificity }=\mathrm{TN} /(\mathrm{TN}+\mathrm{FP}) \\
& \mathrm{PPA}=\mathrm{TP} /(\mathrm{TP}+\mathrm{FP})
\end{aligned}
$$

TABLE (1): Sensitivity, specificity, and PPA results before and after fuzzy enhancement technique.

\begin{tabular}{|c|c|c|}
\hline $\begin{array}{c}\text { Computed } \\
\text { Metric }\end{array}$ & Before fuzzy & After Fuzzy \\
\hline Sensitivity & 80.09 & 96 \\
\hline specificity & 90.4 & 98.1 \\
\hline PPA & 89.47 & 97.95 \\
\hline
\end{tabular}

The fuzzy enhancement technique is implemented for 60 retinal images. The important image processing measurements of entropy, edge intensity, and average gradient are determined for the proposed framework. The quality of the image is improved after the fuzzy enhancement technique. Retinal images enhanced obviously for normal and abnormal images. The entropy, edge intensity, and average gradient are improved after employing the fuzzy enhancement technique with detection of ROI exudates edges. Tables 2 show samples results of the determined edge intensity, average gradient, and entropy before and after fuzzy enhancement. From all presented subjective and objective results, it is noticed the effect of the proposed framework with employing the fuzzy enhancement technique for improving the performance of exudates detection and removal. 
TABLE(2): samples results of the determined edge intensity, average gradient, and entropy before and after fuzzy enhancement.

\begin{tabular}{|c|c|c|c|c|c|}
\hline \multicolumn{2}{|c|}{ Edge Intensity } & \multicolumn{2}{c|}{ Average Gradient } & \multicolumn{2}{c|}{ Entropy } \\
\hline $\begin{array}{c}\text { Before } \\
\text { Fuzzy }\end{array}$ & $\begin{array}{c}\text { After } \\
\text { Fuzzy }\end{array}$ & $\begin{array}{c}\text { Before } \\
\text { Fuzzy }\end{array}$ & $\begin{array}{c}\text { After } \\
\text { Fuzzy }\end{array}$ & $\begin{array}{c}\text { Before } \\
\text { Fuzzy }\end{array}$ & $\begin{array}{c}\text { After } \\
\text { Fuzzy }\end{array}$ \\
\hline 19.627 & 26.1744 & 1.9210 & 2.5355 & 0.2174 & 0.3700 \\
\hline 18.1506 & 25.3762 & 1.7904 & 2.4909 & 0.1944 & 0.3123 \\
\hline 22.247 & 32.0512 & 2.1491 & 3.0508 & 0.2380 & 0.3834 \\
\hline 17.471 & 36.3890 & 1.7162 & 3.5625 & 0.1821 & 0.3150 \\
\hline 21.5573 & 34.2382 & 2.0605 & 3.2615 & 0.1929 & 0.3109 \\
\hline
\end{tabular}

\section{CONCLUSION}

Diabetic maculopathy results from the prolonged diabetes retinopathy that causes blindness. Fuzzy enhancement, morphological operations are used for processing macula in retinal images. The results show that the entropy, average gradient, and edge intensity have been improved with employing the fuzzy enhancement technique for improving the results of exudates detection and removal. The proposed framework facilitates the detection of exudates and identification of the maculpathy disease. The histogram Gaussian gradient is also used for helping to detect the exudates of normal and abnormal cases.

\section{REFERENCES}

[1] Nayak, J., P. S. Bhat,P.S.,Acharya, U. R., "Automatic identification of diabetic maculopathy stages.

[2] Osareh, A., Mirmehdi, M., Thomas, B., and Markham, R., "Automatic recognition of exudative maculopathy using fuzzy Cmeans clustering and neural network".Available on http://www.cs.bris.ac.uk/Publications/ Papers/1000553.pdf , on November 7, 2017.

[3] Vimala and Kajamohideen, Detection of diabetic maculpathy in human retinal images using morphological operations" Online 14 (3) 2014. (http://www.thescipub.com/ojbs.toc), Online Journal of Biological Sciences 14 (3): 175-180, 2014.

[4] Nayak, J., Bhat, P. S., \& Acharya, U. R. (2009). Automatic identification of diabetic maculopathy stages using fundus images. Journal of medical engineering \& technology, 33(2), 119-129.

[5] D. Marin , M. E. Gegundez-Arias, B. Ponte,"An exudate detection method for diagnosis risk of diabetic macular edema in retinal images using feature-based and supervised classification", Medical \& Biological Engineering \& Computing,Jan 2018.

[6] Walter, T., and Klein, J.-C., "Segmentation of color fundus images of the human retina: Detection of the optic disc and the vascular tree using morphological techniques", Springer-Verlag Berlin Heidelberg 2001, pp. 282-287.

[7] HashikinK., \& Isa, N. A. M. (2012, March). Enhancement of the low contrast image using fuzzy set theory. In Computer Modelling and Simulation (UKSim), 2012 UKSim 14th International Conference on (pp. 371-376). IEEE.

[8] Akhavan, R., \& Faez, K. (2013, December). Automated retinal blood vessel segmentation using fuzzy mathematical morphology and morphological reconstruction. In International Symposium on Artificial Intelligence and Signal Processing (pp. 131-140). Springer, Cham.

[9] Li, G., Tong, Y., \& Xiao, X. (2011). Adaptive fuzzy enhancement algorithm of surface image based on local discrimination via grey entropy. Procedia Engineering, 15, 1590-1594.

[10] Cheng, H. D., \& Xu, H. (2000). A novel fuzzy logic approach to contrast enhancement. Pattern recognition, 33(5), 809-8.

[11] Chaira, T. (2015). Medical image processing: Advanced fuzzy set theoretic techniques. CRC Press.
[12] Wang, X. Y., Wang, T., \& Bu, J. (2011). Color image segmentation using pixel wise support vector machine classification. Pattern Recognition, 44(4), 777-787.

[13] ] Dong-liang, P., \& An-ke, X. (2005, October). Degraded image enhancement with applications in robot vision. In Systms, Man and Cybernetics, 2005 IEEE International Conference on (Vol. 2, pp. 1837-1842). IEEE .

[14] Kerre, E. E., \& Nachtegael, M. (Eds.). (2013). Fuzzy techniques in image processing (Vol. 52). Physica.

[15] Pal, S. K., \& King, R. (1981). Image enhancement using smoothing with fuzzy sets. IEEE TRANS. SYS., MAN, AND CYBER., 11(7), 494-500.

[16] Morphological

Operations. http://www.viz.tamu.edu/faculty/parkeends489f00/notes/sec1_9.htm 1./ accessed on 7-11-2017.

[17] Shih, F. Y. (2009). Image processing and mathematical morphology: fundamentals and applications. CRC press.

[18] Zhang, X., \& Fan, G. (2006, November). Retinal spot lesion detection using adaptive multiscale morphological processing. In International Symposium on Visual Computing (pp. 490-501). Springer, Berlin, Heidelberg.

[19] McAndrew, A. (2004). An introduction to digital image processing with matlab notes for $\mathrm{scm} 2511$ image processing. School of Computer Science and Mathematics, Victoria Univ. of Tech., 264(1).

[20] Sinthanayothin, C., Boyce, J. F., Cook, H. L., \& Williamson, T. H. (1999). Automated localisation of the optic disc, fovea, and retinal blood vessels from digital colour fundus images. British Journal of Ophthalmology, 83(8), 902-910.

[21] Mui Hong Ang. " Computer -Based Identification of Diabetic Maculpathy Stages Using Fundus Images", Multi-Modality State-ofthe-Art Medical Image Segmentation and Registration Methodologies,2011.

[22] Walter, T., Klein, J. C., Massin, P., \& Erginay, A. (2002). A contribution of image processing to the diagnosis of diabetic retinopathy-detection of exudates in color fundus images of the human retina. IEEE transactions on medical imaging, 21(10), 12361243.

[23] Tang, H., Wu, E. X., Ma, Q. Y., Gallagher, D., Perera, G. M., \& Zhuang, T. (2000). MRI brain image segmentation by multiresolution edge detection and region selection. Computerized Medical Imaging and Graphics, 24(6), 349-357.

[24] Chandy, D. A., \& Kumari, V. V. (2006). Genetic algorithm-based location of optic disc in retinal images. Academic Open Internet Journal, 17.

[25] Singh, J., \& Sivaswamy, J. (2008, February). Fundus foveal localization based on image relative subtraction-IReS approach. In Proceedings of the 14th national conference on communications.

[26] Smith, M. A., \& Kanade, T. (1998, January). Video skimming and characterization through the combination of image and language understanding. In Content-Based Access of Image and Video Database, 1998. Proceedings., 1998 IEEE International Workshop on (pp. 61-70). IEEE.

[27] Lindeberg, T. (2013). Scale selection properties of generalizedscalespace interest point detectors. Journal of Mathematical Imaging and vision, 46(2), 177-210. 